Ce manuscrit est une préimpression qui a été soumise à la revue «The Journal of Quality in Education » This manuscript is a preprint that has been submitted to "The Journal of Quality in Education"

\title{
Psychologie et science ouverte en Afrique : pourquoi est-elle nécessaire et comment peut-on l'implanter?
}

\section{Psychology and open science in Africa: Why is it needed and how can we implement it?}

Auteurs : Soufian Azouaghe ${ }^{12}$, Adeyemi Adetula ${ }^{2}$, Patrick Forscher ${ }^{2}$, Dana Basnight Brown ${ }^{3}$, Nihal Ouherrou ${ }^{4}$, Abdelilah Charyate ${ }^{5}$, Hans IJzerman ${ }^{2}$

Institutions de rattachement : ${ }^{1}$ Université Mohammed V de Rabat, ${ }^{2}$ LIP/PC2S, Université Grenoble Alpes, ${ }^{3}$ United States International University-Africa, ${ }^{4}$ Université Paul Valéry Montpellier 3, ${ }^{5}$ Université Ibn Tofail.

\section{Notes :}

La préparation de ce manuscrit a été financée en partie par une subvention dans le cadre du programme « Investissements d'avenir » (ANR-15-IDEX-02) accordée à Hans Ijzerman.

Coordonnées. Soufian Azouaghe : Département de Psychologie, Faculté des Lettres et des Sciences de Rabat. azouaghe.soufian@gmail.com. Adeyemi Adetula : LIP/PC2S, Université Grenoble Alpes, adeyemi.adetula@univ-grenoble-alpes.fr

Contributions des auteurs. Soufian Azouaghe et Adeyemi Adetula : rédaction et écriture de plusieurs ébauches du présent article. Patrick Forscher, Dana Basnight Brown, Nihal Ouherrou et Abdelilah Charyate : feedbacks et commentaires sur la première et la dernière ébauche du présent article. Hans IJzerman : réécriture des ébauches du présent article plusieurs fois \& commentaires et feedbacks. 


\title{
Résumé
}

La qualité de la recherche scientifique s'évalue non seulement par son impact positif sur le développement socio-économique et le bien-être humain, mais également par sa contribution à l'élaboration des connaissances scientifiques valides et fiables. De ce fait, les chercheurs, quelle que soit leur discipline scientifique, sont censés adopter des pratiques de recherche basées sur la transparence et la rigueur. Or l'histoire des sciences et la littérature scientifique nous apprennent qu'une part importante des résultats scientifiques n'est pas reproductible de façon systématique (Ioannidis, 2005) ${ }^{1}$. Cela renvoie à ce qui est communément connu sous le nom de la «crise de réplication » qui concerne aussi bien les sciences de la nature que les sciences humaines et sociales, dont la psychologie ne fait pas exception.

Dans cet article, nous présentons d'abord certains aspects de la crise de réplication et les pratiques de recherche douteuses. Puis, nous abordons la manière dont nous pouvons impliquer plus de laboratoires en Afrique dans la recherche scientifique mondiale, notamment le Psychological Science Accelerator (PSA). Dans ce sens, nous développerons un tutoriel pour les laboratoires en Afrique, en mettant en avant les pratiques de la science ouverte. Enfin, nous discutons de comment rendre psychologie scientifique plus participative et inclusive.

Mots-clés : Crise de Réplication, Science Ouverte, Révolution de Crédibilité, Psychological Science Accelerator, Recherche Africaine.

\begin{abstract}
The quality of scientific research is assessed not only by its positive impact on socio-economic development and human well-being, but also by its contribution to the development of valid and reliable scientific knowledge. Thus, researchers regardless of their scientific discipline, are supposed to adopt research practices based on transparency and rigor. However, the history of science and the scientific literature teach us that a part of scientific results is not systematically reproducible (Ioannidis, 2005). This is what is commonly known as the "replication crisis" which concerns the natural sciences as well as the social sciences, of which psychology is no exception.
\end{abstract}

Firstly, we aim to address some aspects of the replication crisis and Questionable Research Practices (QRPs). Secondly, we discuss how we can involve more labs in Africa to take part in the global research process, especially the Psychological Science Accelerator (PSA). For these goals, we will develop a tutorial for the labs in Africa, by highlighting the open science practices. In addition, we emphasize that it is substantial to identify African labs needs and factors that hinder their participating in the PSA, and the support needed from the Western world. Finally, we discuss how to make psychological science more participatory and inclusive.

Keys words: Replication Crisis, Open Science, Credibility Revolution, Psychological Science Accelerator, African Research. 


\section{Introduction}

La qualité de la recherche fondamentale est généralement évaluée par son niveau de contribution à produire des connaissances scientifiques valides et fiables. Aussi, les chercheurs, dans le monde qu'en Afrique, sont soucieux de l'impact positif que la science peut générer au niveau du développement socio-économique et du bien-être humain. Un tel impact ne peut se réaliser que si la production scientifique est suffisamment fiable, transparente et accessible. Hélas les vingt dernières années ont révélé que la base de nos connaissances scientifiques n'est ni assez fiable ni assez accessible (surtout du point de vue africain) tel que nous le souhaiterions. Ces problèmes menacent la capacité et le rôle crucial de la connaissance scientifique dans le développement de la société et la qualité de vie de la population, et par conséquent ils devront être placés au cœur des préoccupations et priorités des chercheurs africains.

Dans cet article, nous présentons autant que faire se peut les problèmes liés à la fiabilité et à l'accessibilité des connaissances scientifiques en psychologie, du moins tels qu'ils sont manifestés en Amérique du Nord et en Europe. Nous décrivons ensuite un ensemble d'initiatives appelées «mouvement de la science ouverte », en anglais «open science movement » qui ont pour but d'améliorer la crédibilité de la recherche scientifique en la rendant plus transparente et plus accessible. Enfin, nous proposons un tutoriel qui décrit la manière dont les chercheurs africains peuvent s'impliquer dans la science ouverte, en l'occurrence les initiatives et les projets de collaboration à grande échelle en vue d'assurer une recherche scientifique hautement fiable et transparente. Le but à terme est de permettre à l'ensemble de chercheurs et d'acteurs scientifiques en Afrique de participer activement au mouvement de la science ouverte.

Dans ce contexte, il faut noter que la participation au mouvement de la science ouverte peut générer des avantages considérables non seulement pour les chercheurs africains, mais aussi pour les pays dans lesquels ils travaillent, notamment que nombreux d'entre eux s'appuient encore sur des pratiques de recherche dites «standard research practices ». Ainsi, il n'est pas clair dans quelle mesure les résultats et les conclusions des études menées par les chercheurs africains sont reproductibles. Quoi qu'il en soit, l'engagement et l'implication dans la pratique de la science ouverte doivent être motivés et animés, entre autres, par le fait que les connaissances scientifiques sont souvent en libre accès ; un précieux avantage pour les chercheurs disposant de ressources logistiques et financières limitées. 


\section{Pourquoi les chercheurs africains devraient-ils adopter les pratiques de la science ouverte?}

$\mathrm{Au}$ cours des dix ou vingt dernières années (selon le point de vue de chacun), les scientifiques en sont venus à remettre en question la stabilité, la reproductibilité et la fiabilité des résultats scientifiques (e.g., Doyen, Klein, Pichon, \& Cleeremans, 2012² ; Pashler, Rohrer, \& Harris, $2013^{3}$ ). Pour de nombreux chercheurs en psychologie, l'année 2011 est peut-être la plus mémorable, durant laquelle une importante affaire de fraude s'est produite et la discussion à propos des analyses de données problématiques «data contingent analyses » a été mise en avant (Rodgers \& Shrout, 2018) ${ }^{4}$. L'exemple le plus emblématique qui illustre ces analyses problématiques est l'article de Daryl Bem $(2011)^{5}$ publié dans la prestigieuse revue : «Journal of Personality and Social Psychology ». Bem, un psychologue social éminent et reconnu, avait mené une série d'expériences dans lesquelles il "prouvait" que les gens peuvent voir l'avenir (see the futur). Ces idées étaient étayées par des preuves statistiques généralement acceptées à l'époque et suscitaient ainsi la curiosité des chercheurs de reproduire les résultats obtenus par Bem. Les études menées par la suite ont montré une série d'effets nuls convaincants, contredisant directement l'idée selon laquelle les gens peuvent prévoir l'avenir (pour plus de détails, voir Francis, $2012^{6}$; Galak, LeBouef, Nelson, \& Simmons, $2012^{7}$; Schimmack, 2012 ${ }^{8}$ ).

Depuis 2011, la communauté scientifique a mené des études de réplication multi-sites sur de grands échantillons. Une grande partie de ces études de réplication n'a pas trouvé les mêmes effets que ceux des résultats originaux, qui ont souvent été publiés dans des revues prestigieuses à fort impact. Par exemple, Klein et al. (2014) ${ }^{9}$ ont réussi à répliquer 10 des 13 résultats. L’Open Science Collaboration $(2015)^{10}$ a répliqué avec succès 36 des 97 . Camerer et al. (2016) ${ }^{11}$ ont pu répliquer 11 des 18. Ebersole et al. $(2016)^{12}$ ont réussi à répliquer 4 des 10. Cova et al. $(2018)^{13}$ ont répliqué avec succès 29 des 37. Camerer et al. (2018) ${ }^{14}$ ont réussi à reproduire 13 effets sur $21(62 \%)$. Klein et al. (2018) ${ }^{15}$ ont répliqué avec succès 14 des 28.

Ces éléments nous laissent affirmer qu'il y a une crise. À ce titre, plusieurs auteurs ont, au cours des dix dernières années, décrit les dangers que présentent les analyses statistiques problématiques pour la fiabilité de la recherche scientifique. Plus précisément, Simmons, Nelson et Simonsohn $(2011)^{16}$ ont mené deux études et simulations afin de démontrer à quel point il est facile de trouver des résultats significatifs lorsque l'effet nul ne devrait pas être rejeté (erreur de type I). Plus encore, ils ont souligné qu'ils pouvaient démontrer qu'après avoir écouté la chanson «When I'm Sixty-Four » des Beatles, les participants rajeunissaient deux ans (une idée si absurde bien évidemment). En utilisant donc ce qu'ils appellent des pratiques de 
recherche flexibles «flexible research practices », il est plausible d'obtenir des valeurs $\mathrm{p}$ significatives pour toute idée aussi absurde soit-elle (voir également, John, Loewenstein, \& Prelec, 2012) ${ }^{17}$. Ces pratiques douteuses ou questionnables « questionnable research practices » sont l'une des raisons principales de l'échec des réplications. Quelles sont donc certaines de ces pratiques de recherche douteuses ? Les chercheurs africains commettent-ils les mêmes erreurs que celles des chercheurs européens et américains ? Y a-t-il d'autres raisons pour lesquelles les résultats scientifiques sont de qualité insuffisante?

En effet, les pratiques de recherche douteuses regroupent un ensemble de techniques et d'usages qui permettent d'obtenir des résultats significatifs, même si l'hypothèse nulle est vraie. Parmi ces pratiques figure le HARKing «Hypothesizing After the Results are Known » qui désigne le fait d'émettre des hypothèses après que les résultats soient connus. Autrement dit, le HARKing consiste à présenter une hypothèse post hoc ou a posteriori (c'est-à-dire après avoir vu les résultats) comme si c'était formulée a priori (Kerr, 1998) ${ }^{18}$. À cela s'ajoutent la suppression d'une condition expérimentale, le « optional stopping » qui correspond à la poursuite de l'analyse des données jusqu'à ce que la valeur $\mathrm{p}$ soit significative, la suppression des participants, la suppression des items d'un questionnaire après avoir vu les résultats pour que les effets soient significatifs, et ainsi de suite. Les pratiques de recherche douteuses englobent donc toutes les méthodes qui augmentent les chances d'obtenir un effet significatif après avoir analysé les données et pris connaissance des résultats.

Outre les analyses statistiques problématiques que nous venons de mentionner, il y a d'autres raisons qui peuvent expliquer le manque ou la non reproductibilité des résultats scientifiques. Il s'agit par exemple du fait de ne pas signaler les résultats non significatifs (file drawering) qui représente une menace majeure à la reproductibilité. Dans ce cadre et au fil des années, il y a lieu de noter que les résultats dans la littérature psychologique étaient extrêmement positifs, c'est-à-dire significatifs (Sterling, Rosenbaum, \& Weinkam, 1995) ${ }^{19}$. Dans ce sens, des études ont révélé que la littérature psychologique était l'un des domaines qui rapportait le plus grand pourcentage de résultats positifs, avec environ $91.5 \%$ des résultats apportés pour soutenir les hypothèses des chercheurs (Fanelli, 2010) ${ }^{20}$. Cela est statistiquement impossible dans la mesure où la puissance moyenne (la chance de détecter un effet lorsqu'il existe) dans la littérature psychologique est de 0.23 pour un petit effet, 0.60 pour un effet moyen et 0.78 pour un grand effet (Szucs \& Ioannidis, 2017) ${ }^{21}$.

Dans ce contexte et face à ces problèmes, un nouveau format d'article scientifique nommé «Registered Reports » ou rapport enregistré est apparu. Un format selon lequel les données ne 
sont collectées qu'après un peer review de l'introduction théorique, de la méthode et des analyses statistiques prévues. Une fois accepté, l'article est publié même si les résultats sont négatifs. À ce sujet, Scheel, Schijen et Lakens $(2020)^{22}$ ont récemment comparé les résultats dans la littérature psychologique des articles standards «standard reports » et les Registered Reports. Ils ont constaté que dans les articles standards, $96 \%$ des résultats sont positifs comparativement à $44 \%$ pour les Registered Reports. Il en ressort donc que le biais de publication «publication bias » représente l'un des facteurs fondamentaux qui expliquent l'échec des réplications.

Que pouvons-nous, en tant que chercheurs, faire pour freiner ces tendances et ces pratiques ? Au cours des deux dernières décennies, les chercheurs en psychologie ont amorcé une réforme et ouvert une voie prometteuse pour améliorer et changer les pratiques de recherche dans le cadre du mouvement de la science ouverte. Un mouvement qui a déployé des efforts considérables et réalisé des progrès notables en matière de prévention des problèmes de la crise de réplication, en adoptant de nouvelles pratiques visant à rendre la recherche plus ouverte à la vérification et, surtout, plus reproductible et par extension plus accessible. Mais où en est l'Afrique par rapport à tout cela ?

Malgré quelques efforts consentis ici et là, force est de constater, lorsque l'on examine de plus près les projets de réplication internationaux qui ont été réalisés jusque-là, que les pays africains y sont faiblement représentés. Par exemple, dans le Many Labs 1 (Klein et al., 2014) ${ }^{9}$, aucun pays africain n'y figure. En ce qui concerne le Many Labs 2 (Klein et al., 2018) ${ }^{15}$, sur 36 pays et territoires et 79 échantillons, l'Afrique était représentée seulement par 3 pays avec un total de 6 échantillons : Nigeria $(n=1)$, Afrique du Sud $(n=3)$ et Tanzanie $(n=2)$. Il en est de même pour une vaste étude collaborative (et non une étude de réplication) menée par le Psychological Science Accelerator ${ }^{1}$ (Jones et al., under review) ${ }^{23}$ et réalisée dans 11 régions du monde et 41 pays. Sur ces 41 pays, seuls trois pays étaient africains : Kenya, Nigeria et Afrique du Sud.

Bien que cette faible implication des laboratoires africains soulève des questions qui demeurent sans réponse, elle sollicite néanmoins les chercheurs africains pour rattraper ce retard et rejoindre le mouvement de la science ouverte. À ce sujet, il semble opportun de multiplier les efforts pour informer et sensibiliser les acteurs concernés (e.g., enseignants-chercheurs, doctorants et étudiants, organismes de recherche et universités d'Afrique, d'Amérique du Nord

\footnotetext{
${ }^{1}$ Cet article est un registered report dont le titre est : To Which World Regions Does the Valence-Dominance Model of Social Perception Apply?
} 
et d'Europe) en vue de contribuer à l'avancement de la psychologie scientifique selon les nouveaux standards de recherche.

Par ailleurs, diverses raisons peuvent expliquer cette faible représentation des pays africains. Il se peut, à titre d'illustration, que les chercheurs africains ne soient pas au courant de ces projets de réplication. Il s'agit ici d'un manque d'accès (ou à la méconnaissance) aux réseaux de recherche menant ces études, aux revues dans lesquelles les études originales sont publiées, et/ou ils peuvent tout simplement ne pas avoir accès aux réplications publiées.

Étant donné que les chercheurs africains s'appuient en grande partie sur les mêmes procédures de recherche que celles utilisées par les chercheurs d'Amérique du Nord et d'Europe, il est fortement plausible que la recherche africaine souffre aussi du problème de la nonreproductibilité des résultats. En tant que chercheurs africains, nous devons donc prendre conscience de ces réalités et réaliser que la transition vers une science ouverte et l'adoption de nouvelles pratiques de recherche sont la clé pour produire des connaissances et faire des découvertes scientifiques fiables et crédibles.

Si les problèmes de réplication en Afrique peuvent être majoritairement analogues à ceux auxquels les chercheurs d'Amérique du Nord et d'Europe sont confrontés, il faut toutefois noter que les chercheurs africains font face à d'autres problèmes très pesants et plus importants que « la simple » crise de réplication. Beaucoup d'entre nous affrontent d'énormes obstacles en termes de ressources humaines et financières, d'accès à la littérature publiée, de formation limitée et/ou du manque de connaissances techniques sur les méthodes et les analyses statistiques sophistiquées. À cela s'ajoute la méconnaissance des plateformes dédiées à la science ouverte, car nous sommes généralement peu représentés dans les projets collaboratifs internationaux. Dans cette lignée, nous décrivons tour à tour quelques initiatives relatives à la science ouverte lancées en Amérique du Nord et en Europe, en mettant notamment en exergue ce qu'elles pourraient apporter pour la recherche et les chercheurs africains.

\section{Comment les chercheurs africains peuvent-ils être impliqués dans les pratiques de la} science ouverte ? En quoi consiste le mouvement de la science ouverte ?

Il existe plusieurs initiatives susceptibles d'apporter de précieux avantages aux chercheurs africains et auxquelles ils peuvent prendre part. Mais avant de les passer en revue, il convient d'abord d'examiner ce que représente le mouvement de la science ouverte. En effet, une partie de ce mouvement est constituée d'organisations qui offrent et assurent une infrastructure libre, dans le but de rendre possible la pratique de la science ouverte. Le Center 
for Open Science (COS ; https://cos.io) représente l'un des exemples les plus remarquables. Le $\mathrm{COS}$, une organisation à but non lucratif, est un pionnier de la réforme du fonctionnement de la recherche scientifique. Plus particulièrement, le COS consacre d'énormes efforts pour accroître l'ouverture, l'intégrité et la reproductibilité de la recherche en offrant plusieurs services :

- offrir et maintenir une infrastructure libre et ouverte (Open Science Framework, OSF) afin d'assurer la gestion et l'archivage de la documentation scientifique (e.g., préenregistrement, partage du matériel et des données) ;

- soutenir les réseaux scientifiques et encourager le développement de diverses initiatives visant à améliorer la reproductibilité de la recherche scientifique (e.g., open science collaboration, projets Many Labs, projets Many Analysts);

- offrir une variété de services de formation sur les nouvelles pratiques de recherche, les nouveaux outils ainsi que les techniques statistiques.

Il existe en outre plusieurs infrastructures à accès ouvert comme la Plateforme de la Science Ouverte (Open Science Framework, https://osf.io), GitHub (https://github.com), Dataverse (https://dataverse.org), Figshare (https://figshare.com). Ces infrastructures et plateformes ont pour but commun la valorisation et la promotion de la science ouverte (e.g., archiver, organiser, partager, collaborer).

Parmi les initiatives auxquelles les chercheurs africains peuvent participer figurent les Many Labs. C'est une initiative soutenue par le COS qui constitue un excellent projet de collaboration à l'échelle internationale. Toutefois, à l'heure actuelle, il n'est pas certain que de nouveaux projets de réplication Many Labs seront lancés. Cependant, il existe une autre option supplémentaire qui est le Psychological Science Accelerator (PSA). Un réseau impliquant un nombre conséquent de laboratoires localisés dans de nombreux pays (plus de 500 laboratoires, représentant plus de 70 pays) auquel les chercheurs africains peuvent adhérer et participer. Ce réseau a été créé pour permettre et soutenir des projets de recherche collaboratifs, c'est-à-dire des études scientifiques de grande envergure menées dans plusieurs sites et laboratoires. La mission du PSA est ainsi de créer une plateforme qui accélère l'accumulation de données fiables et généralisables en psychologie, en coordonnant la collecte de données pour des études sélectionnées démocratiquement et impartialement (Moshontz et al., 2018) ${ }^{24}$.

Il y a lieu de préciser que le PSA vise à couvrir une large diversité culturelle et géographique aussi bien au niveau des chercheurs qu'au niveau des échantillons et des thèmes de recherche. Pour ce faire, elle adopte une politique de recherche fondée sur la diversité et l'inclusion, le 
pouvoir décentralisé, la transparence, la rigueur et l'ouverture à la critique (Moshontz et al., 2018 $)^{24}$. L'inscription au PSA se fait à travers le lien suivant : https://psysciacc.org/newmember-registration. La soumission d'une proposition d'un projet de recherche au PSA est aussi possible, en suivant un processus spécifique qui se déroule en plusieurs étapes : soumission et évaluation de la proposition, préparation et mise en œuvre de la collecte des données, analyse des données et diffusion des résultats (pour plus de détails, voir Moshontz et al., 2018) ${ }^{24}$.

Une autre initiative qui mérite également d'être mentionnée est le projet de réplication et d'éducation collaborative (Collaborative Réplications and Education Project, CREP). Ce projet offre un cadre pertinent aux étudiants de premier cycle pour participer activement à la conduite de réplication directe de haute qualité. Il s'inscrit entièrement dans une visée éducative, c'est-à-dire enseigner aux étudiants les bonnes pratiques de recherche, en réalisant des réplications directes des travaux très cités dans la littérature et en appliquant un ensemble de méthodes de la science ouverte (Wagge et al., 2019) ${ }^{25}$. Pour s'y inscrire, il faut aller sur le lien suivant : https://osf.io/wfc6u. En outre, il importe de préciser que ces initiatives de crowdsourcing proposent des avantages précieux, dans la mesure où elles permettent aux chercheurs, d'une part de participer à la science ouverte, et d'autre part leur enseigner les nouvelles pratiques de recherche (e.g., pré-enregistrement, partage des données, publication des préprints en ligne).

Une fois que les chercheurs se sont familiarisés avec les pratiques de la science ouverte, ils peuvent se lancer dans leurs propres projets de recherche, en menant leur propre étude de réplication, en effectuant un pré-enregistrement ou en soumettant un Registered Report. De nos jours, les études de réplication sont souvent acceptées par les revues scientifiques. À cet égard, il y a lieu de distinguer deux types de réplication : la réplication directe et la réplication conceptuelle. La réplication directe ou exacte consiste à utiliser le même matériel, la même procédure expérimentale, les mêmes variables dépendantes et le même type de participants que ceux de l'étude originale. En revanche, la réplication conceptuelle vise à tester la même hypothèse que celle de l'étude originale, mais en introduisant des changements qui peuvent concerner l'opérationnalisation du phénomène, les variables indépendantes et dépendantes, les participants, etc. (Crandall \& Sherman, 2016) ${ }^{26}$.

Dans cette veine, les chercheurs s'appuient actuellement sur le pré-enregistrement ou le Registered Reports pour réaliser une réplication. Pour le pré-enregistrement, les chercheurs fournissent, avant la conduite de l'étude, une description précise d'un certain nombre 
d'éléments à savoir les hypothèses, la méthodologie (e.g., conception, caractéristiques des participants, technique d'échantillonnage, taille d'échantillon fixée a priori) et les analyses statistiques prévues (script préparé avant la collecte des données). Ces éléments sont stockés dans une plateforme en ligne accessible qui permet à la communauté scientifique de faire les vérifications nécessaires s'il y a besoin.

En ce qui concerne les Registered Reports, ils sont très similaires au pré-enregistrement. Cependant, la principale différence réside dans le fait que l'étude est soumise un peer review, et ce, avant que la collecte des données soit effectuée (Chambers, $2013^{27}$; Nosek \& Lakens, $2014^{28}$ ). Pour être plus précis, les Registered Reports suivent un processus de peer review qui se déroule en deux phases. Dans la première phase, les chercheurs soumettent le protocole de l'étude à une revue (liste des revues disponible sur https://cos.io/rr/), en soulignant les hypothèses, la méthode et les analyses des données à utiliser et, si la proposition est retenue, il reçoit une acceptation préliminaire (IPA pour In Principle Acceptance). Cet examen par les pairs permet aux chercheurs d'obtenir des commentaires avant la collecte des données et la revue - si l'IPA est fournie - s'engage à publier l'article quels que soient les résultats. Dans la seconde phase, les chercheurs soumettent le manuscrit final avec les résultats et la discussion qui fait l'objet d'une évaluation par les paires, notamment pour vérifier la concordance entre la version initiale et la version finale et accepté même si les résultats ne sont pas significatifs.

Outre la rigueur et la transparence qu'assurent le pré-enregistrement et les Registered Reports, ces pratiques permettent également aux chercheurs de distinguer clairement entre deux types de recherche empirique, et ce, avant la collecte des données : la recherche exploratoire versus la recherche confirmatoire. En effet, la recherche exploratoire ne part pas d'hypothèses solides a priori et consiste donc à répondre à des questions de recherche relativement ouvertes. En d'autres mots, la recherche exploratoire sert à développer ou affiner des hypothèses qui seront ultérieurement mises à l'épreuve dans le cadre d'une étude (recherche) confirmatoire. Celle-ci consiste en revanche à répondre à une question de recherche bien fondée théoriquement et vérifier une hypothèse prédéfinie et clairement énoncée.

En résumé, il convient de noter que la transition vers la science ouverte est en train de se faire doucement en psychologie, notamment dans les pays occidentaux. Ainsi, les initiatives et les pratiques de recherche que nous venons de décrire ont certainement donné et donnent encore des résultats encourageants pour la psychologie scientifique, mais le continent africain n'a en quelque sorte pas participé à ce grand chantier. D'où l'intérêt d'aborder maintenant comment la science ouverte et les pratiques qui en dépendent peuvent être implantés en Afrique. 


\section{Comment implanter les pratiques de la science ouverte en Afrique ?}

$\mathrm{Nul}$ besoin de rappeler que nous sommes tous d'accord sur l'importance d'implanter et mettre en œuvre la science ouverte en Afrique. À ce sujet, il importe de poser trois questions qui sont à notre sens fondamentales afin d'appréhender comment la pratique de la science ouverte peut être inculquée dans la recherche psychologique en Afrique.

\subsection{Dans quelle mesure les psychologues et chercheurs africains adhèrent-ils aux principes de la science ouverte ?}

Cette question rend directement compte de l'intérêt de former les chercheurs africains sur les pratiques de la science ouverte. Comme nous l'avons discuté plus haut, les nouvelles pratiques de recherche font partie des usages et des stratégies en matière de conception et de conduite de la recherche scientifique, et ce, dans différents laboratoires et centres qui sont pleinement investis dans le mouvement de la science ouverte en vue de rendre la production et la connaissance scientifiques vérifiables et accessibles.

Dans cette lignée, il s'avère primordial que les chercheurs africains soient convaincus, aujourd'hui plus que jamais, de l'intérêt de changer la façon dont la recherche scientifique est menée, en gardant à l'esprit le fait que la production de résultats scientifiques ne suffit pas, dans le sens où ces résultats devraient surtout être ouverts à la vérification par les paires. Autrement dit, il faut qu'il ait un partage de la littérature, de la méthodologie de recherche suivie étape par étape, des données et des résultats afin que les autres aient accès à toutes les ressources nécessaires pour pouvoir reconduire ou répliquer l'étude originale. Cela nous rendra tout au moins rigoureux et offrira à d'autres chercheurs la possibilité de nous fournir des commentaires constructifs et contribuer à la discussion scientifique autour de nos thèmes de recherche. Par conséquent, le partage de ces informations sur les études scientifiques que nous conduisons contribuera amplement à éviter de s'engager dans le P-Hacking, le HARKing, le file drawering, et par conséquent il permettra un peer review de haute qualité et une production scientifique hautement fiable.

\subsection{Comment impliquer plus de laboratoires en Afrique?}

L'objectif de cette section est de discuter de comment impliquer les chercheurs africains dans les projets collaboratifs en accédant aux laboratoires et aux réseaux de recherche scientifiques internationaux, en l'occurrence le PSA. Pour y parvenir, deux catégories d'objectifs, distinctes mais complémentaires, méritent d'être pris en considération. 


\subsubsection{Objectifs à court terme}

Cette catégorie d'objectifs vise à créer un impact immédiat pour faire implanter la science ouverte en Afrique ainsi qu'impliquer les chercheurs africains dans des projets de recherche internationaux. Pour ce faire, il nous semble fondamental de :

- participer à la collecte de données en tant que chercheurs africains dans le cadre des projets de recherche et de collaboration PSA ;

- fournir des tutoriels en ligne sur les pratiques de la science ouverte ;

- organiser des événements scientifiques en Afrique (e.g., congrès, conférences et ateliers) sur la crise de réplication et les pratiques de la science ouverte ;

- pouvoir accéder à la documentation scientifique ouverte, aux revues scientifiques ouvertes ainsi qu'aux manuels et logiciels ;

- permettre à l'Afrique de collaborer et de participer activement à la recherche scientifique (c'est-à-dire d'être également celle qui statue sur les questions de recherche à étudier) ;

- prévoir des prix des ambassadeurs pour des projets scientifiques ouverts et collaboratifs tels que le COS et le PSA.

\subsubsection{Objectifs à long terme}

Cette catégorie d'objectifs a pour but d'inculquer les principes et la culture de la science ouverte en vue de garantir la durabilité des gains à court terme. Elle inclut ainsi :

- appréhender les valeurs et saisir les cultures locales ainsi que les limites des chercheurs africains pour établir un terrain d'entente qui ne compromettra pas nécessairement les principes de la science ouverte ;

- développer des échelles de mesure et des inventaires faciles à utiliser et validés pour la population africaine, notamment en ce qui a trait à la recherche expérimentale ;

- insister sur l'éthique et l'intégrité de la recherche scientifique pour se prémunir de cas de la fraude scientifique à savoir la falsification et la fabrication des données et des résultats ;

- connaître (et peut-être adopter) des méthodes et des pratiques de recherche appropriées (e.g., techniques d'échantillonnage, conception). Il s'agit en quelque sorte de créer des méthodes et des pratiques de recherche adaptées pour la psychologie africaine ;

- équiper les laboratoires du matériel nécessaire pour mener des expériences selon les principes et les exigences de la science ouverte. Cela encouragera les enseignants, les chercheurs et les étudiants intéressés à effectuer des séjours ou des stages scientifiques dans des laboratoires optant pour les pratiques de la science ouverte ;

- créer des revues scientifiques en ligne en libre accès. 


\subsection{Comment pouvons-nous rendre la psychologie scientifique plus inclusive?}

Bien que la psychologie ne soit pas encore reconnue à sa juste valeur scientifique et contributive dans un grand nombre de pays africains, les chercheurs et psychologues africains doivent continuer leurs efforts pour promouvoir la psychologie scientifique. À cet égard, les chercheurs dans les pays du monde, où la psychologie a une histoire longue et glorieuse ainsi qu'elle bénéficie d'une large visibilité tant au niveau institutionnel que social, peuvent jouer un rôle prépondérant dans l'accompagnement de leurs homologues africains tout au long du processus de la transition vers la science ouverte. Le but à terme est de permettre aux chercheurs africains de prendre le relais pour asseoir et édifier une psychologie scientifique qui promeut les nouvelles pratique de recherche et la culture de la science ouverte.

L'implication des laboratoires africains dans la recherche psychologique internationale peut être profondément ancrée dans la formation et la mise en œuvre d'une politique inclusive et participative. Dans ce cadre, l'analyse SWOT (Strengths, Weaknesses, Opportunities, Threats) de la recherche scientifique en Afrique se propose comme une clé fondamentale pour mettre en place ladite politique. En effet, la recherche en Afrique a ses propres particularités, limites et forces que nous devons prendre en considération lorsque nous discutons de l'inclusion des chercheurs africains.

Le financement de la recherche en Afrique et les divers problèmes qui en résultent sont également une problématique majeure dont il faut tenir compte. En effet, nul besoin de noter que les chercheurs africains ont beaucoup de difficultés à trouver des financements pour mener à bien leurs travaux de recherche. En absence ou à cause du manque de subventions et de ressources financières, il est d'usage que, dans la plupart des cas, les chercheurs africains payent une maison d'édition pour publier leurs recherches. À cela s'ajoutent le coût d'achat des échelles et des inventaires, la réalisation de l'analyse des données, l'accès à Internet, le coût de l'emploi d'assistants de recherche, l'administration des tests et des inventaires, la réalisation d'expériences, etc. C'est pour ces raisons et non des moindres que nous proposons une solution basée sur une politique qui comprend, entre autres, l'accès gratuit aux ressources documentaires telles que : https://info.africarxiv.org, https://psyarxiv.com, l'acquisition gratuite des échelles de mesure, la publication gratuite, etc.

Une autre façon de permettre aux chercheurs africains de s'impliquer dans la science ouverte est ce que nous appelons « amener le laboratoire à eux, Take the Lab to them ». Il s'agit de créer des laboratoires qui répondent aux exigences minimales en termes de personnel et de moyens pour qu'ils puissent fonctionner correctement. De même, les laboratoires déjà existants sont à 
encourager à pratiquer la philosophie de la science ouverte. Bien que cette philosophie puisse être « étrange » et nouvelle pour les chercheurs africains, il devrait avoir un terrain d'entente qui permet de la mettre en place de façon douce et progressive. Le but est non seulement d'instaurer une politique durable, mais aussi adaptée aux pratiques locales et aux principes de la science ouverte pour la psychologie africaine.

L'une des principales pistes qui peuvent favoriser l'inclusion de l'Afrique dans la science ouverte est la collaboration. Celle-ci peut se concrétiser ainsi : échange d'idées et de ressources, congé sabbatique dans les deux sens (Afrique-Europe/Amérique du Nord), co-écriture d'articles, établissement de partenariats de recherche, collaboration avec les associations africaines de psychologie, etc.

Par ailleurs, il s'avère important de concevoir une politique relative à la formation des formateurs. C'est probablement l'un des meilleurs moyens pour impliquer les chercheurs africains dans le projet de la science ouverte dans la perspective de créer une tradition en la matière. De ce fait, la mise en place d'un programme complet pour les personnes intéressées, notamment celles originaires des pays africains, facilitera directement ou indirectement l'acceptation de la science ouverte et l'inclusion de l'Afrique dans la transition vers les nouvelles pratiques de recherche. Ces personnes formées constitueront un précieux moyen de transmission et du transfert du savoir, de la culture et des principes de la science ouverte. De même, leur savoir-faire et technique seront très utiles pour le fonctionnement et le développement des laboratoires africains.

\section{Conclusion}

Le présent article a pour objectif d'attirer l'attention des chercheurs africains sur la « révolution » méthodologique et scientifique qui est en train de se réaliser en psychologie au niveau mondial. Une révolution qui marque la rupture avec les pratiques de recherche douteuses et la transition vers les pratiques de la science ouverte. Il décrit en outre les avantages inestimables offerts par le mouvement de la science ouverte, notamment le Psychological Science Accelerator. En effet, le PSA offre aux chercheurs africains l'occasion de mener des recherches au niveau international en utilisant les pratiques et les outils de la science ouverte, et par conséquent produire des connaissances scientifiques plus transparentes et plus crédibles. Il est temps, aujourd'hui plus que jamais, d'instaurer une psychologie scientifique ouverte en Afrique. C'est une véritable et prometteuse opportunité à saisir ainsi qu'une grande entreprise scientifique à laquelle les chercheurs et psychologues africains peuvent entièrement s'engager et amplement contribuer. 


\section{Bibliographie}

${ }^{[1]}$ Ioannidis, J. P. A. (2005).Why most published research findings are false. PLoS Med, 2(8), e124. doi: 10.1371/journal.pmed.0020124.

${ }^{[2]}$ Doyen, S., Klein, O., Pichon, C. L., \& Cleeremans, A. (2012). Behavioral Priming: It's All in the Mind, but Whose Mind? PLoS ONE, 7(1): e29081. doi: 10.1371/journal.pone.0029081.

${ }^{[3]}$ Pashler, H., Rohrer, D., \& Harris, C. (2013). Can the goal of honesty be primed? Journal of Experimental Social Psychology, 49, 959-964. doi : 10.1016/j.jesp.2013.05.011.

[4] Rodgers, J. L., \& Shrout, P. E. (2018). Psychology's replication crisis as scientific opportunity: A précis for policymakers. Policy Insights from the Behavioral and Brain Sciences, 5(1), 134-141. doi: 10.1177/2372732217749254.

${ }^{[5]}$ Bem, D. J. (2011). Feeling the future: Experimental evidence for anomalous retroactive influences on cognition and affect. Journal of Personality and Social Psychology, 100(3), 407-425. doi: 10.1037/a0021524.

${ }^{[6]}$ Francis, G. (2012). Too good to be true: Publication bias in two prominent studies from experimental psychology. Psychonomic Bulletin \& Review, 19, 151-156. doi: 10.3758/s13423-012-0227-9.

${ }^{[7]}$ Galak, J., LeBoeuf, R. A., Nelson, L. D., \& Simmons, J. P. (2012). Correcting the past: Failures to replicate psi. Journal of Personality and Social Psychology, 103(6), 933-948. doi:10.1037/a0029709.

${ }^{[8]}$ Schimmack, U. (2012). The ironic effect of significant results on the credibility of multiplestudy articles. Psychological Methods, 17(4), 551-566. doi: 10.1037/a0029487.

${ }^{[9]}$ Klein, R. A., Ratliff, K. A., Vianello, M., Adams, R. B., Jr., Bahník, Š., Bernstein, M. J., . . . Nosek, B. A. (2014). Investigating variation in replicability: A "Many Labs" replication project. Social Psychology, 45, 142-152. doi:10.1027/1864-9335/a000178.

[10] Open Science Collaboration. (2015). Estimating the reproducibility of psychological science. Science, 349(6251). doi: 10.1126/science.aac4716.

${ }^{[11]}$ Camerer, C. F., Dreber, A., Forsell, E., Ho, T.-H., Huber, J., Johannesson, M., . . W Wu, H. (2016). Evaluating replicability of laboratory experiments in economics. Science, 351, 1433-1436. doi:10.1126/science.aaf0918.

${ }^{[12]}$ Ebersole, C. R., Atherton, O. E., Belanger, A. L., Skulborstad, H. M., Allen, J. M., Banks, J. B., . . Nosek, B. A. (2016). Many Labs 3: Evaluating participant pool quality across the academic semester via replication. Journal of Experimental Social Psychology, 67, 68-82. doi:10.1016/j .jesp.2015.10.012.

${ }^{[13]}$ Cova, F., Strickland, B., Abatista, A., Allard, A., Andow, J., Attie, M., . . . Zhou, X. (2018). Estimating the reproducibility of experimental philosophy. Review of Philosophy and Psychology, 1-36. doi:10.1007/s13164-018-0400-9.

${ }^{[14]}$ Camerer, C. F., Dreber, A., Holzmeister, F., Ho, T.-H., Huber, J., Johannesson, M., . . Wu, H. (2018). Evaluating the replicability of social science experiments in Nature and Science between 2010 and 2015. Nature Human Behaviour, 2, 637-644. doi:10.1038/s41562-018-0399-z. 
${ }^{[15]}$ Klein, R. A., Vianello, M., Hasselman, F., Adams, B. G., Adams, R. B., Alper, S., . . Nosek, B. A. (2018). Many Labs 2: Investigating variation in replicability across sample and setting. Advances in Methods and Practices in Psychological Science, 1(4), 443-490, doi: $10.1177 / 2515245918810225$.

[16] Simmons, J.P., Nelson, L.D., \& Simonsohn, U. (2011).False-positive psychology: undisclosed flexibility in data collection and analysis allows presenting anything as significant. Psychological Science, 22, 1359-1366. doi: 10.1177/095679761141 7632.

${ }^{[17]}$ John, L. K., Loewenstein, G., \& Prelec, D. (2012). Measuring the prevalence of questionable research practices with incentives for truth telling. Psychological Science, 23, 524-532. doi: 10.1177/0956797611430953.

${ }^{[18]}$ Kerr, N. L. (1998). HARKing: Hypothesizing After the Results are Known. Personality and Social Psychology Review, 2(3), 196-217. doi: 10.1207/s15327957pspr0203_4.

[19] Sterling, T. D., Rosenbaum, W. L., \& Weinkam, J. J. (1995), "Publication decisions revisited: The effect of the outcome of statistical tests on the decision to publish and vice versa. The American Statistician, 49, 108- 112.

${ }^{\text {[20] }}$ Fanelli, D. (2010). "Positive'" results increase down the hierarchy of the sciences. Plos One, 5(3), e10068. doi:10.1371/journal.pone.0010068.

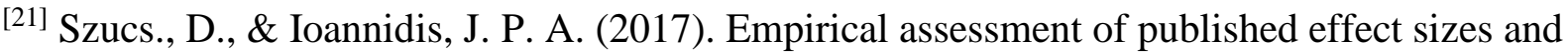
power in the recent cognitive neuroscience and psychology literature. PLoS Biol, 15(3): e2000797. https://doi.org/10.1371/journal.pbio.2000797.

${ }^{[22]}$ Scheel, A. M., Schijen, M., \& Lakens, D. (2020). An excess of positive results: Comparing the standard Psychology literature with Registered Reports. PsyArXiv.

${ }^{[23]}$ Jones, B., DeBruine, L., Flake, J., Liuzaa, M. T., Antfolk, J., Arinze, N., ... Coles, N. (underreview). To Which World Regions Does the Valence-Dominance Model of Social Perception Apply? (PSA001; Registered Report Stage 2). Nature Human Behaviour.

${ }^{[24]}$ Moshontz, H., Campbell, L., Ebersole, C., IJzerman, H., Urry, H. L., Forscher, P. L., ... Chartier, C. R. -2018). The Psychological Science Accelerator: Advancing Psychology Through a Distributed Collaborative Network. Advances in Methods and Practices in Psychological Science, 1(4) 501-515. doi: 10.1177/2515245918797607.

${ }^{[25]}$ Wagge, J. R., Brandt, M. J., Lazarevic, L. B., Legate, N., Christopherson, C., Wiggins, B., \& Grahe, J. E. (2019). Publishing research with undergraduate students via replication work: The collaborative replications and education project. Frontiers in Psychology, 10, 247.

[26] Crandall, C. S., \& Sherman, J. W. (2016). On the scientific superiority of conceptual replications for scientific progress. Journal of Experimental Social Psychology, 26, 9399. doi: 10.1016/j.jesp.2015.10.002.

[27] Chambers, C. D. (2013). Registered reports: A new publishing initiative at Cortex. Cortex, 49, 609-610.

${ }^{[28]}$ Nosek, B. A., \& Lakens, D. (2014). Registered reports: A method to increase the credibility of published results. Social Psychology, 45(3), 137-141. doi: 10.1027/18649335/a000192. 